\title{
Grain bulk density measurement based on wireless network
}

\author{
Fangming Wu, Bingfang $\mathrm{Wu}^{\mathrm{a}}$ and Leidong Yang \\ Key Laboratory of Digital Earth Sciences, Institute of Remote Sensing and Digital Earth, Chinese Academy of \\ Sciences, 100101, Beijing, China
}

\begin{abstract}
To know the accurate quantity of stored grain, grain density sensors must be used to measure the grain's bulk density. However, multi-sensors should be inserted into the storage facility, to quickly collect data during the inventory checking of stored grain. In this study, the ZigBee and Wi-Fi coexistence network's ability to transmit data collected by density sensors was investigated. A system consisting of six sensor nodes, six router nodes, one gateway and one Android Pad was assembled to measure the grain's bulk density and calculate its quantity. The CC2530 chip with ZigBee technology was considered as the core of the information processing, and wireless nodes detection in sensor, and router nodes. ZigBee worked in difference signal channel with Wi-Fi to avoid interferences and connected with Wi-Fi module by UART serial communications interfaces in gateway. The Android Pad received the measured data through the gateway and processed this data to calculate quantity. The system enabled multi-point and real-time parameter detection inside the grain storage. Results show that the system has characteristics of good expansibility, networking flexibility and convenience.
\end{abstract}

\section{Introduction}

Inventory checking of grain storages expends a great deal of manpower and material resources in China, but the accuracy of measurement results is still not satisfying, and the actual density of the grain storages are difficult to determine. Sensors offer a potential solution to this underlying problem. The grain bulk density sensor was designed according to the relationship between permittivity and bulk density of grain [1], and the electromagnetic wave transmission method was used to estimate grain permittivity, and then calculated bulk density.

A wireless network is any type of communication network that uses wireless data connections for connecting network nodes thus, obviates the costly process of introducing cables into a building [2]. ZigBee is a low-cost, low-power, wireless mesh network standard targeted at the wide development of long battery life devices in wireless control and monitoring applications [3]. ZigBee devices can transmit data over long distances by passing data through a mesh network of intermediate devices to reach more distant ones, whereas $\mathrm{Wi}-\mathrm{Fi}$ is a wireless local area network based on IEEE 802.11 standards [4]. Devices which can use Wi-Fi technology includes personal computers, smartphones, tablet computers and digital cameras.

Decision-makers need accurate information on amount and quality of stored grain to prevent storage losses, which can be a major hindrance to food security. Grain bulk density sensors can be

\footnotetext{
${ }^{\mathrm{a}}$ Corresponding author : wubf@radi.ac.cn
} 
used to measure grain bulk density, which is a good indicator of stored grain conditions. However, multi-sensors should be inserted into the grain storage to quickly collect measured data during the inventory checking of stored grain. This can be costly and sometimes give erroneous results. Therefore, in this study, the ZigBee and Wi-Fi coexistence network's ability to transmit data collected by density sensors was investigated.

\section{System design}

\subsection{System architecture}

A wireless network system was developed and it consisted of six sensor nodes, six router nodes, one gateway and one Android Pad, to measure grain bulk density and calculate quantity as shown in Figure 1. ZigBee network was used for communication among sensor nodes, router nodes and gateway. The Android Pad connected with the gateway through Wi-Fi to send commands and receive data.

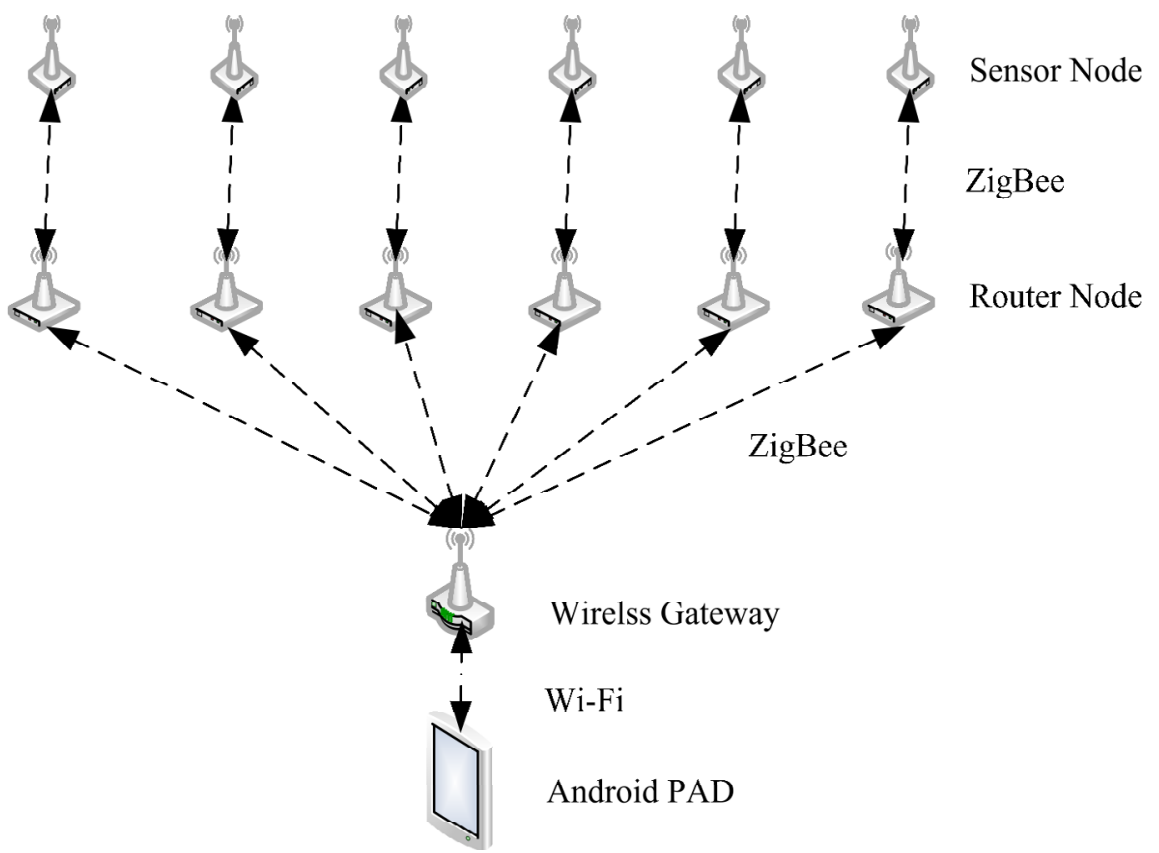

Figure 1. Grain bulk density measurement system architecture.

The sensor nodes received data from the environment and sent it to the router. As well as running an application function, a router node could act as an intermediate router, thereby passing on data from other devices. The gateway had to be set in advance to be able to receive data from sensor or router nodes.

To communicate and transmit data wirelessly, each sensor or router node required a ZigBee module. In addition, to be able to use the entire module is used, such devices require additional power from the battery.

\subsection{System components}

The sensor node consisted of a CC2530 ZigBee module, AD8302 phase detector, AD8532 operation amplifier and TPS60211 power manager. The CC2530 is a true system-on-chip (SOC) solution for 
ZigBee applications. The CC2530 combined the excellent performance of a leading RF transceiver with an industry-standard enhanced $8051 \mathrm{MCU}$ [5].

The router node consisted of a CC2530 ZigBee module, AD9851 Direct Digital Synthesizer (DDS) and MAX2650 amplifier. The shortwave signal was generated by an AD9851, and controlled by a CC2530 ZigBee module through the SPI interface.

The wireless gateway consisted of a CC2530 ZigBee module and a USR-WIFI232 Wi-Fi module. ZigBee worked in different signal channels with Wi-Fi to avoid interferences and was connected to the Wi-Fi module through the UART serial communications interfaces in gateway.

The hardware specifications of the system used in this study are shown in Table 1;

Table 1. System hardware specifications.

\begin{tabular}{|c|c|c|}
\hline SN. & Hardware & Specifications \\
\hline 1 & $\begin{array}{l}\text { sensor } \\
\text { node }\end{array}$ & $\begin{array}{c}\text { CC2530 ZigBee module } \\
\text { AD8302 phase detector } \\
\text { AD8532 operation amplifier } \\
\text { TPS60211 power manager }\end{array}$ \\
\hline 2 & router node & $\begin{array}{c}\text { CC2530 ZigBee module, } \\
\text { AD9851 DDS } \\
\text { MAX2650 amplifier }\end{array}$ \\
\hline 3 & $\begin{array}{l}\text { wireless } \\
\text { gateway }\end{array}$ & $\begin{array}{c}\text { CC2530 ZigBee module } \\
\text { USR-WIFI232 Wi-Fi module }\end{array}$ \\
\hline 4 & $\begin{array}{l}\text { Android } \\
\text { Pad }\end{array}$ & $\begin{array}{c}\text { CPU: Marvell PXA986 } \\
\text { GPU : PowerVR SGX540 } \\
\text { RAM :1GB } \\
\text { Flash ROM 8GB } \\
\text { OS :Android v4.1.2 (Jelly Bean }\end{array}$ \\
\hline
\end{tabular}

\subsection{Wireless network configuration}

The Gateway had a user interface for system configuration called Manager System, which is similar to configuration application on routers in general. By default, Manager System could be accessed using a web browser via WiFi (using 10.10.100.254). The initial view of Manager System appeared shortly after logging in with correct credentials.

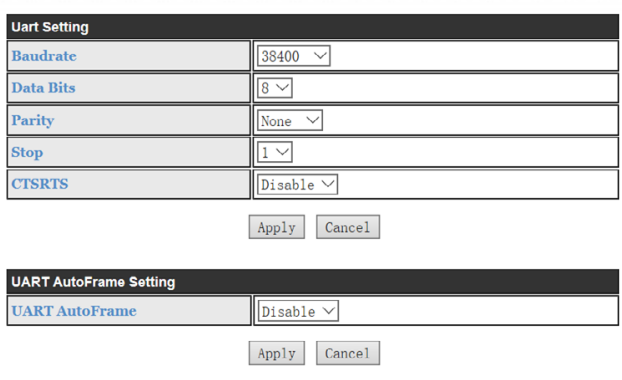

Figure 2. Gateway UART Settings.

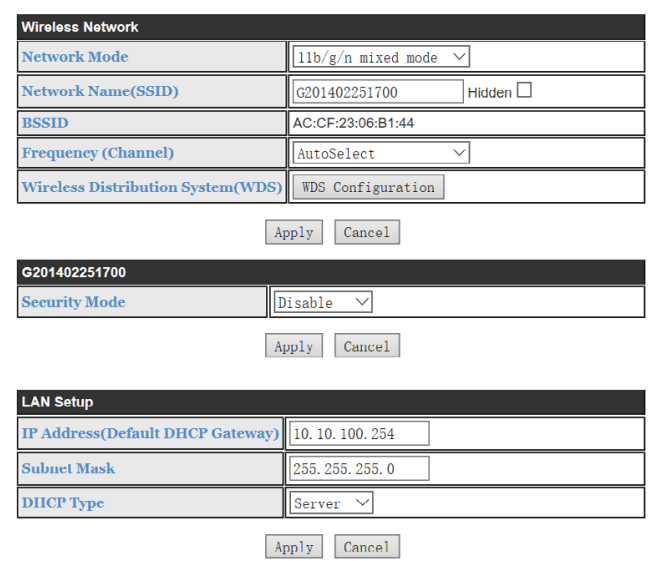

Figure 3. Gateway Network Configuration. 
The pre-requisite UART settings included the Bandrate, Data Bits and Stop Bit (Fig. 2). Furthermore, network configuration such as Network Name (SSID) and Channel were performed in the interfaces tab in the Manager System, as shown in Fig. 3.

\section{Experiment result}

In the study, four sensor nodes and four router nodes connected to a wireless gateway were used. The Gateway was then connected to an Android Pad via a Wi-Fi network. The resultant network topology is shown in Figure 4.

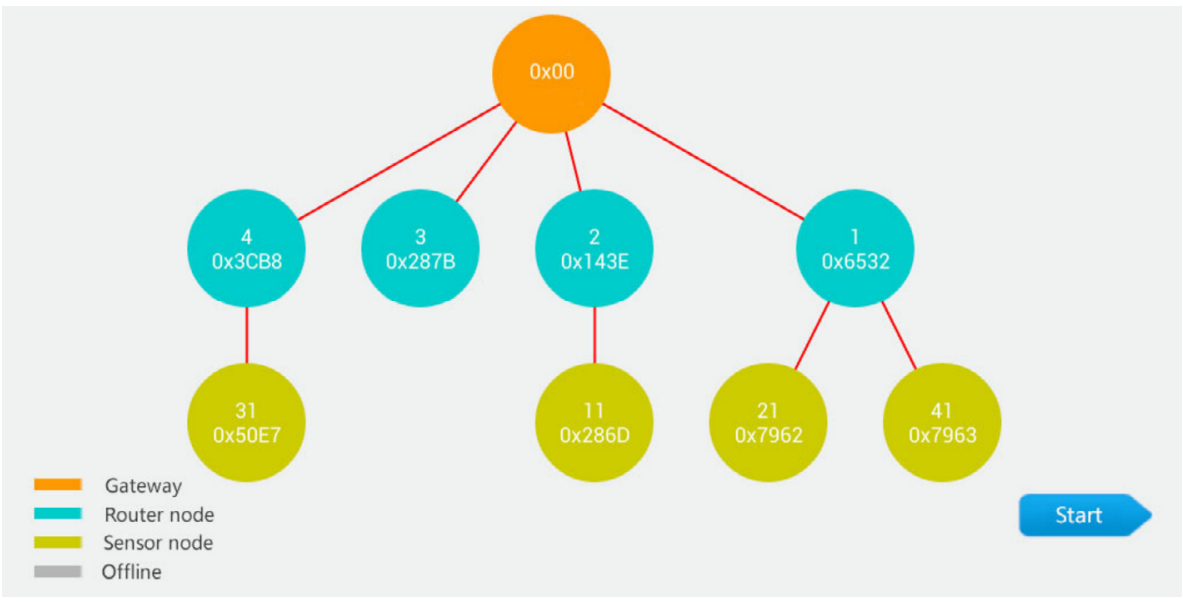

Figure 4. Network topology in this study.

The short address of gateway was $0 x 00$, and each sensor node or router node had a different short address. Pressing the "Start" button was done to receive measured data after all devices were connected to the gateway.

The Android Pad received measured data from each sensor node through the gateway and processed this data to calculate quantity. The result of the measurement is given in Figure 5.

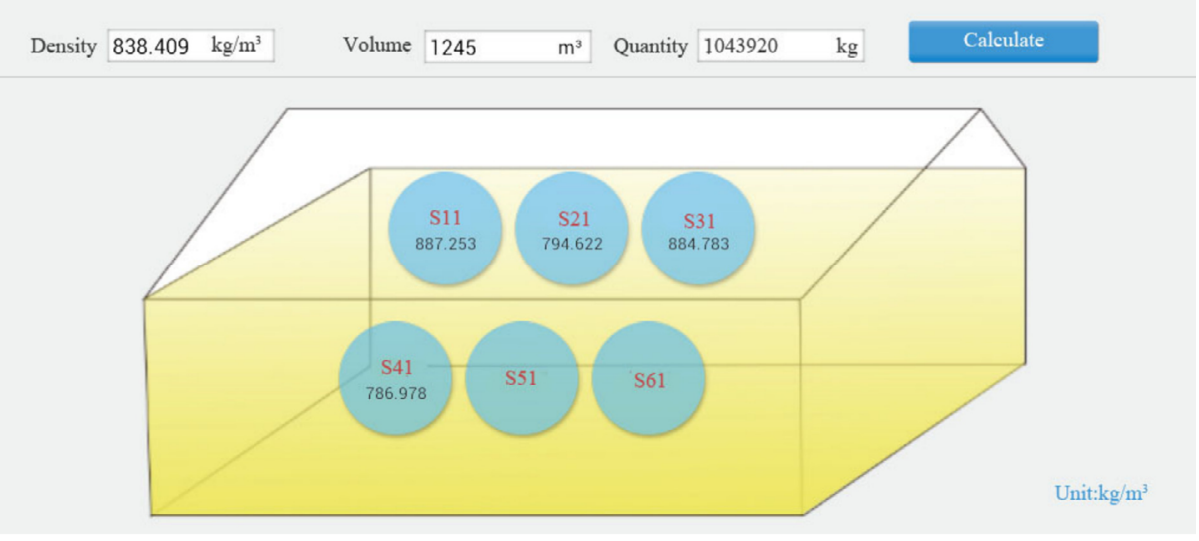

Figure 5. Result of the measurement.

The Android Pad had a MySQL local database to store the values of the measurement. A local database can be very vulnerable to damage and memory limitations of storage, so it is advisable to back-up the data to an external storage. 


\section{Conclusion}

A grain bulk density measurement system based on wireless network was developed in this study. It reliably proves that Wi-Fi network and ZigBee network can be used together. By joining networks, an Android Pad can be employed to send commands, receive data and manage measured values. The system performed multi-point and real-time detection inside the grain storage. These results show that this system has the characteristics of good expansibility, networking flexibility and convenience. It is recommended, however, to explore the potential for using 4G/3G/GPRS to remotely monitor the grain bulk density in future.

\section{Acknowledgements}

This research was supported by the Special Fund for Grain-Scientific Research in the Public Interest under Project 201413003-6.

\section{References}

1. K. Sacilik and A. Colak, Powder Technology, 203, 365-370 (2010)

2. G. Miao, J. Zander, K.W. Sung, and B. Slimane, Fundation of Mobile Data Networks (2016)

3. What is ZigBee? The Zigbee Alliance, http://www.zigbee.org/what-is-zigbee/

4. What is Wi-Fi(IEEE 802.11x)? Webopedia Definition, http://www.webopedia.com/TERM/W/Wi_Fi.html 